\title{
Charging of Thin Film Phase Plates under Electron Beam Irradiation
}

\author{
Marek Malac ${ }^{1,2}$, Marco Beleggia ${ }^{4}$, Ray Egerton ${ }^{1,2}$, Masahiro Kawasaki ${ }^{3}$, Michael Berge ${ }^{1}$, Yoshio \\ Okura $^{3}$, Isamu Ishikawa ${ }^{3}$, Kohei Motoki ${ }^{3}$ \\ 1. National Institute of Nanotechnology, 11421 Saskatchewan Drive, Edmonton, Canada \\ 2. Department of Physics, University of Alberta, T6G 2E1, Edmonton, Canada. \\ 3. JEOL Ltd. 1-2 Musashino- 3 chome, Akishima, Tokyo 198-8558, Japan \\ 4. Center for Electron Nanoscopy, Technical University of Denmark, Denmark.
}

Electrostatic charging of samples under electron beam irradiation in a TEM affects the observed contrast [1]. It can also be used to produce Zernike-like phase contrast when a suitable uniform thin film is placed in the back focal plane of the objective lens, referred to as a hole-free phase plate (HFPP) contrast [2]. Here we report the effect of heating and biasing on contrast transfer function. Our results may be relevant to sample charging and other implementations of thin-film type phase plates.

An arrangement similar to [2,3] was used for the measurements: a uniform thin film of Ge or $\mathrm{C}$ was placed at a selected area aperture plane that was conjugate to the back focal plane of the objective lens [2], shown in Fig. 1. Rather than grounded, the HFPP thin film was biased up to $\pm 27 \mathrm{~V}$ relative to ground. The HFPP was also heated to $\sim 200{ }^{\circ} \mathrm{C}$. A series of images was recorded with $\sim 1.6 \mathrm{~A} / \mathrm{cm}^{2}$ current density at the HFPP in 19 s (i.e. $30 \mathrm{C} / \mathrm{cm}^{2}$ ) interval between images. The contrast transfer function (CTF) was evaluated by locating the positions of extrema in a radially averaged power spectrum (PS) of the images [2]. The position of the extrema allowed us to measure the phase shift of the HFPP [2]. Fig. 2 shows that the PS initially changes fast (black), but settles towards a steady state (in blue) with the first maximum in the PS far more intense and at lower spatial frequencies than in the initial PS (black). The increased intensity in the low spatial frequencies $(q)$ in the steady state PS corresponds to good contrast transfer of low $q$ in the HFPP set up [2]. Fig. 3 shows the effect of heating on carbon HFPP. It appears that HFPP settling at $200{ }^{\circ} \mathrm{C}$ reaches a plateau in less than $100 \mathrm{~s}$ (red solid symbols) as opposed to room temperature (RT) experiment (blue empty symbols) where $\sim 285 \mathrm{~s}$ is needed. While the resistivity of amorphous carbon (or Ge) is expected to decrease with $\mathrm{T}$, a $\sim 3 \mathrm{x}$ decrease of the HFPP settling-time with $\Delta \mathrm{T}=180{ }^{\circ} \mathrm{C}$ is at odds with the small temperature coefficients, suggesting electron tunneling charge transport or semiconducting behavior. At $200{ }^{\circ} \mathrm{C}$ the initial plateau is followed by an increase of the $q$ at which the extremes is located. This indicates a second mechanism that is slower than the initial one starts to dominate and reaches a plateau after $\sim 330 \mathrm{~s}$. It is likely that the initial (fast) settling mechanism is related to charge redistribution in the HFPP while the second, slower, mechanism could involve be diffusion of species adsorbed at the surface of the HFPP into the edges of the beam. Unlike a typical redistribution of contamination, the $200{ }^{\circ} \mathrm{C}$ data reach saturation. At room temperature the surface diffusion rates are much slower than at $200{ }^{\circ} \mathrm{C}$ reducing the effect on the CTF. Fig. 4 shows carbon HFPP settling in two different experiments (shown in blue and red respectively). The profiles were obtained by subtracting a polynomial fit from log (CTF) similar to Fig.1. The initial (black) profiles correspond to nearly standard bright field TEM imaging. The two color profiles (bright red and bright blue) in the $q<0.15 \mathrm{~nm}^{-1}$ region, where the effect of defocus of the objective lens is small [2], exhibit peaks at same spatial frequencies indicating good reproducibility of the HFPP from experiment to experiment. The effect of bias of the HFPP on position of the extrema tends to be small with slight positive bias slightly improving the transfer at low $q<0.01 \mathrm{~nm}^{-1}$, but the stability of the Ge HFPPs, as judged by the position of high spatial frequency extrema $\left(q>0.15 \mathrm{~nm}^{-1}\right)$ 
improves when a bias is applied. A +9V positive bias to had a similar effect on improving the Ge HFPP stability as heating.

A model, based on the balance between secondary electron emission induced by the primary beam and the compensating current from ground, together with a proper description of the interfaces and contacts, is being developed. The model will provide further insights in amount and distribution of charge on the HFPP and the origin of the effects of heating and bias [4].

\section{References}

[1] J. Berriman and K. L. Leonard, Ultramicroscopy 19 (1986), p. 349.

[2] M. Malac et. al., Ultramicroscopy 118 (2012), p. 77.

[3] K. Danov, R. Danev and K. Nagayama, Ultramicroscopy 87 (2000), p. 45 .

[4] Support of NINT, NSERC and JEOL Ltd. is gratefully acknowledged.

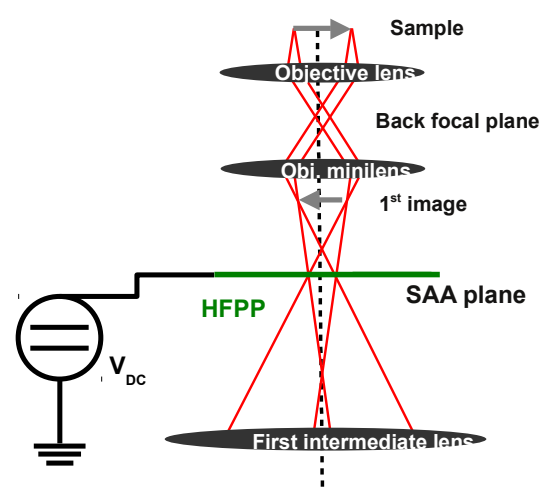

Fig. 1 Experimental set up. The HFPP is placed at a elected area aperture (SAA plane). It is connected to a set of batteries to provide positive or negative bias relative to ground electrode that is common with the instrument ground. The HFPP can be also

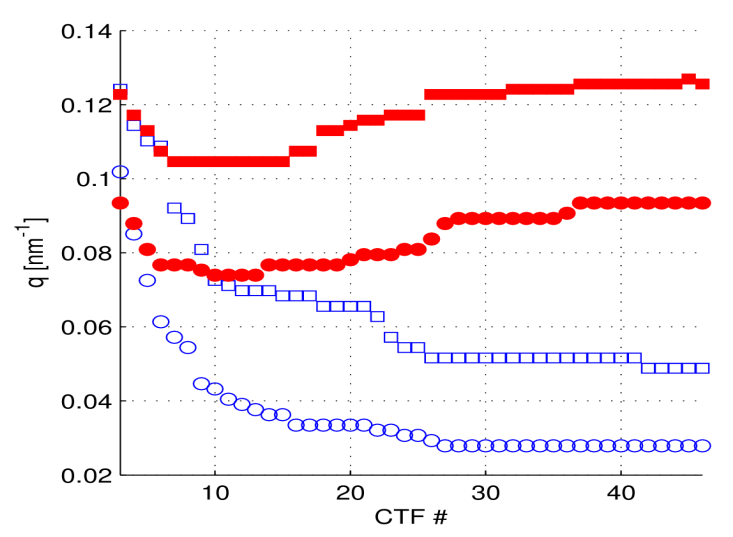

Fig. 3 Settling of carbon HFPP at room temperature (empty blue symbols) and at $200^{\circ} \mathrm{C}$ (solid red symbols). Second minimum (circles) and maximum (squares) is plotted at both temperatures.

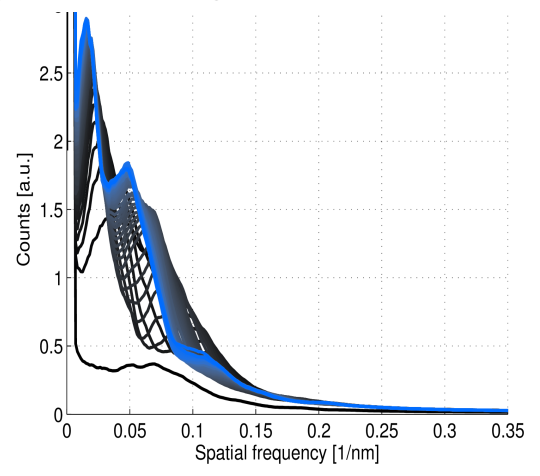

Fig 2. Carbon HFPP settling. Black (lower) to blue (upper) power spectrum corresponds to increase in irradiation time. The HFPP was irradiated with current density $\sim 1.6 \mathrm{~A} / \mathrm{cm}^{2}$ and the subsequent power spectra were collected in about $30 \mathrm{C} / \mathrm{cm}^{2}$ increments. The HFPP settled after $\sim 300 \mathrm{C} / \mathrm{cm}^{2}$ at HFPP.

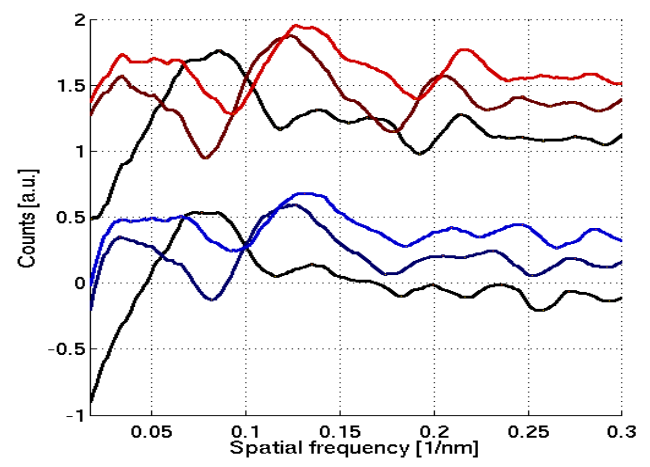

Fig. 4. Reproducibility of carbon HFPP. Three profiles selected from two separate experiments (red and blue) at $200^{\circ} \mathrm{C}$ appear to lead to extrema of the HFPP at the same spatial frequencies after similar irradiation dose was applied. The profiles are offset vertically for clarity. 